\title{
Perspective author's response: Uncompetitive/Fast Off-rate (UFO) mechanism of pathologically- activated neuroprotective drugs
}

\author{
Stuart A. Lipton
}

I thank Professor Manev for his comments relating his work on RADA (receptor abusedependent antagonism) $)^{1}$ in 1990 to my recent article on PAT (pathologically activated therapeutics) $)^{2}$. In point of fact, my recent Perspective mentions that such drugs aimed to decrease overuse of receptors have been around for a quite a while - specifically, work on dilantin and lidocaine dating back at least to the early 1970 s is mentioned. The idea was revisited for glutamate excitotoxicity in our 1988 and 1990 publications $^{3-5}$. However, the mere idea of treating receptor overuse in glutamate excitotoxicity, as brought up previously by our group and by Professor Manev, misses the main point of my recent article: what is important here is the idea, first promulgated by my former graduate student H.-S. Vincent Chen in his 1990 doctoral thesis at Harvard Medical School and subsequently in our 1992 publication $^{6}$, that a unique mechanism of action is needed in order to produce a clinically-tolerated drug to combat excitotoxicity. Our group has recently termed one such mechanism as 'UFO' drugs, representing an Uncompetitive/Fast Off-rate form of inhibition that preferentially blocks increasing (pathological) activity of a receptor, because of the uncompetitive nature of the antagonism, but allows normal activity to continue. For normal synaptic activity to remain relatively unaffected, the drug must have a fast off-rate and therefore not accumulate at the receptor.

Although not mentioned in the comments of Professor Manev, the PAT/UFO strategy has already met with considerable success, as witnessed by the recent clinical approval of the NMDA ( $N$-methyl-D-aspartate)-receptor antagonist memantine by both the Food and Drug Administration (FDA) in the United States and the European Medicines Agency (EMEA) of the European Union. We reported back in 1990 and 1992 that the PAT requirements were met in the case of memantine for excitotoxicty because of its UFO mechanism of open-channel block of NMDA-receptor-associated ion channels ${ }^{6}$.
Thus, memantine blocks excessive (predominantly extrasynaptic) NMDA-receptoroperated channels while relatively sparing normal (synaptic) neurotransmission associated with NMDA-receptor stimulation ${ }^{6-11}$. Our group believes that in order to produce additional clinically-tolerated therapeutic agents, this UFO mechanism of action will become a cornerstone of future drug development, not only for drugs that act in the brain but for other types of pharmaceuticals as well.

Stuart A. Lipton is at the Burnham Center for Neuroscience, Aging, and Stem Cell Research, The Salk Institute, The Scripps Research Institute, and the University of California at San Diego, La Jolla, California 92037, USA.

1. Manev, H., Costa, E., Wroblewski, J. T. \& Guidotti, A. Abusive stimulation of excitatory amino acid receptors: a strategy to limit neurotoxicity. FASEB J. 4 2789-2797 (1990).

2. Lipton, S. A. Pathologically activated therapeutics for neuroprotection. Nature Rev. Neurosci. 8, 803-808 (2007).

3. Karschin, A., Aizenman, E. \& Lipton, S. A. The interaction of agonists and noncompetitive antagonists at the excitatory amino acid receptors in rat retinal ganglion cells in vitro. J. Neurosci. $\mathbf{8}$, 2895-2906 (1988)

4. Hahn, J. S., Aizenman, E. \& Lipton, S. A. Central mammalian neurons resistant to glutamate toxicity are made sensitive by elevated extracellular calcium toxicity blocked by the $N$-methyl-D-aspartate antagonist MK-801. Proc. Natl Acad. Sci. USA 85 6556-6560 (1988).

5. Levy, D. I. \& Lipton, S. A. Comparison of delayed administration of competitive and uncompetitive antagonists in preventing NMDA receptor-mediated neuronal death. Neurology 40, 852-855 (1990).

6. Chen, H.-S. V. et al. Open-channel block of $N$-methyl- D -aspartate (NMDA) responses by memantine: therapeutic advantage against NMDA receptormediated neurotoxicity. J. Neurosci. 12, 4427-4436 (1992).

7. Lipton, S. A. Prospects for clinically-tolerated NMDA antagonists: open-channel blockers and alternative redox states of nitric oxide. Trends Neurosci. 16, 527-532 (1993)

8. Chen, H.-S. V. \& Lipton, S. A. Mechanism of memantine block of NMDA-activated channels in rat retinal ganglion cells: uncompetitive antagonism. J. Physiol. (Lond.) 499, 27-46 (1997).

9. Lipton, S. A. \& Rosenberg, P. A. Mechanisms of disease: excitatory amino acids as a final common pathway for neurologic disorders. N. Engl. J. Med. 330, 613-622 (1994).

10. Chen, H.-S. V. et al. Neuroprotective concentrations of the NMDA open-channel blocker memantine are effective without cytoplasmic vacuolization following post-ischemic administration and do not block maze learning or LTP. Neuroscience 86, 1121-1132 (1998).

11. Chen, H.-S. V. \& Lipton, S. A. The chemical biology of clinically tolerated NMDA receptor antagonists. J. Neurochem. 97, 1611-1626 (2006). 